\title{
A Hybrid Approach to Clinical Guideline and to Basic Medical Knowledge Conformance
}

\author{
A. Bottrighi ${ }^{1}$, F. Chesani ${ }^{2}$, P. Mello ${ }^{2}$, G. Molino ${ }^{3}$, M. Montali ${ }^{2}$, S. Montani ${ }^{1}$, \\ S. Storari ${ }^{4}$, P. Terenziani ${ }^{1}$, and M. Torchio ${ }^{3}$ \\ 1 DI, Univ. del Piemonte Orientale, via Bellini 25/g, 15100 - Alessandria, Italy \\ \{alessio.bottrighi, terenz, stefania\}@mfn.unipm.it \\ 2 DEIS - Univ. di Bologna, viale Risorgimento 2, 40136 - Bologna, Italy \\ \{federico.chesani, paola.mello, marco.montali\}@unibo.it \\ 3 Az. Ospedaliera S. Giovanni Battista, via Bramante 88, Torino, Italy \\ mtorchio@molinette.piemonte.it, giampaolo.molino@virgilio.it \\ ${ }^{4}$ DI - Univ. di Ferrara, via Saragat 1, 44136 - Ferrara, Italy \\ sergio.storari@unife.it
}

\begin{abstract}
Several computer-based approaches to Clinical Guidelines have been developed in the last two decades. However, only recently the community has started to cope with the fact that Clinical Guidelines are just a part of the medical knowledge that physicians have to take into account when treating patients. The procedural knowledge in the guidelines have to be complemented by additional declarative medical knowledge. In this paper, we analyse such an interaction, by studying the conformance problem, defined as evaluating the adherence of a set of performed clinical actions w.r.t. the behaviour recommended by the guideline and by the medical knowledge.
\end{abstract}

\section{Introduction}

Clinical Guidelines are "systematically developed statements to assist practitioner and patient decisions about appropriate health care for specific clinical circumstances" [4]. In the last decade, the research about computerized guidelines has reached a relevant role within the Artificial Intelligence in Medicine community, and many different approaches and projects have been developed to create domain-independent computer-assisted tools for managing, acquiring, representing and executing clinical guidelines [8]. Only recently, however, some approaches have started to consider that Clinical Guidelines (henceforth CG) cannot be interpreted (and executed) "in isolation". CGs are just a part of the medical knowledge that physicians have to take into account when treating patients. The procedural knowledge in the guidelines have to be complemented by additional declarative medical knowledge. In this paper we explore such an interaction from the viewpoint of the conformance problem, intended as the adherence of an observed execution to both types of knowledge. 


\section{Guidelines, Basic Medical Knowledge and Conformance}

Several conditions are usually implicitly assumed by experts building a CG:

(i) ideal patients, i.e., patients that have "just the single" disease considered in the CG (thus excluding the concurrent application of more than one CG), and are "statistically relevant" (they model the typical patient affected by the given disease), not presenting rare peculiarities/side-effects;

(ii) ideal physicians executing the CG, i.e., physicians having the basic medical knowledge, allowing them to properly apply the CGs to specific patients;

(iii) ideal context of execution, so that all necessary resources are available.

Assumption $(i)$ is needed, since the variety of possible patients is potentially infinite, and the CG cannot explicitly cope with all possible nuances and exceptions. Assumption ( $\mathrm{ii}$ ) is also necessary, since the CG focuses on the proper treatment of a specific disease, and can not code all the "basic medical knowledge". Nevertheless, such a knowledge is needed when a CG will be executed, to properly adapt the "general" prescriptions in the CG to the specific case constituted by a particular patient. Finally, experts cannot know all possible execution contexts, so that they usually assume availability of resources (assumption (iii)).

As a consequence of these assumptions, a CG cannot be interpreted as a protocol which has to be applied tout cour, and the actions prescribed by CGs cannot be interpreted as "must do" actions. The intended semantics of CGs cannot be analysed in isolation from the basic medical knowledge. Roughly speaking, given a patient $X$ to which a $\mathrm{CG} \mathcal{G}$ has to be applied in a context $\mathcal{C}, \mathcal{G}$ has to be interpreted as a set of default prescriptions: whenever $X$ and $\mathcal{C}$ fit with $\mathcal{G}$ 's prescriptions, they must be executed. However, $X$ (or $\mathcal{C}$ ) may have peculiar features which are not explicitly covered by $\mathcal{G}$. In such a case, the basic medical knowledge must be considered to identify the correct actions.

The interplays between CG's knowledge and the Basic Medical Knowledge (BMK from now on) can be very complex, as shown by the following examples.

Example 1. Patients suffering from bacterial pneumonia caused by agents sensible to penicillin and to macrolid, allergic to penicillin, must be treated with macrolid.

BMK: Don't administer drug $X$ to a patient if she is allergic to $X$.

In Ex. 1, two alternative treatments (penicillin or macrolid) are possible given the $\mathrm{CG}$, but one of them is excluded, given the underlying basic medical knowledge, because of allergy to penicillin. Here the BMK "complete" the CG and help to discriminate among different alternatives. In other cases, the basic medical knowledge may apparently contradict the recommendations in the CG.

Example 2. Patient with acute myocardial infarction presenting with acute pulmonary edema; before performing coronary angiography it is mandatory to treat the acute heart failure.

BMK: The execution of any CG may be suspended, if a problem threatening the patient's life suddenly arise. Such a problem has to be treated first. 
In Ex. 2 the execution of a CG is suspended, due to the arise of a problem threatening the patient's life. Notice that the "contradiction" (logical inconsistency) between CG's recommendations and BMK is only apparent. It arises just in cases one interpret CG's recommendations as must do, while, as a matter of facts, they may be emended by BMK.

Also, there seems to be no general rule in case of "apparent contradiction": it could be that BMK recommendations "win" over CG ones, or vice versa. In Ex. 3 a treatment is performed even if it may be dangerous for the patient. In some sense, not only some CG's prescriptions are "defeasible", since they may be overridden by BMK, but the same also holds for part of BMK.

Example 3. In a patient affected by unstable angina and advanced predialytic renal failure, coronary angiography remains mandatory, even if the contrast media administration may cause a further final deterioration of the renal functions, leading the patient to dialysis.

When considering conformance of an execution w.r.t. a CG, additional actions not foreseen by the CG could be considered as an issue. This could happen as a consequence of some particular routine (even a CG applied at the same time), like in Ex. 4.

Example 4. Calcemia and glycemia are routinely performed in all patients admitted to the internal medicine ward of Italian hospitals, regardless of the disease.

To summarize, CG semantics is very complex, and cannot be simply interpreted as a strict, normative procedures. The context of execution and the BMK complement the prescriptions in the CGs, bridging (at least in part) the gap between the "ideal" and the "real" application cases. In this hybrid situation the property of conformance, intended as the adherence of an execution to CGs and BMK recommendation, becomes more and more important, and yet difficult to be captured. Defining conformance as the simple conjunction of the conformances to each different piece of knowledge might not be the best choice. Both the CG knowledge and the BMK can be defeated, hence there is no general rule on the prevalence of one or the other. The conformance evaluation is a task that necessarily requires the intervention of a physician. However, such a task can be very difficult and time consuming.

\section{Evaluating Conformance}

We propose to combine, in an hybrid system, tools used for independently evaluating conformance to CGs and BMK. The aim is twofold: on one side, we combine both types of knowledge; on the other side, we facilitate the physician task by identifying discrepancies between actual executions and CG/BMK recommendations, and by suggesting possible explanations. The architecture is shown in Figure 1.

Procedural and workflow-like systems seems to be the most common choice for representing CGs [6], while declarative approaches might be preferable to 


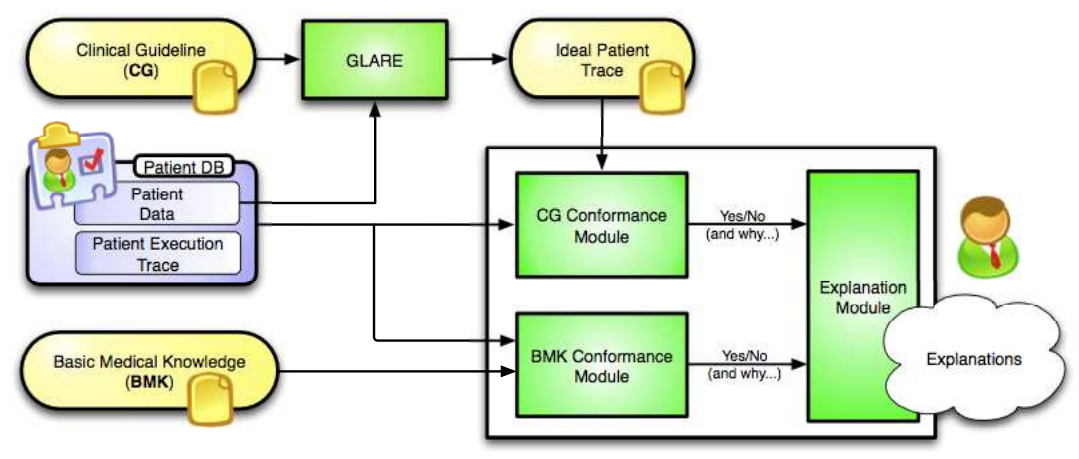

Fig. 1. Architecture of our hybrid approach for the conformance evaluation

represent BMK, due to its similarity to knowledge bases. Our system takes as input three different pieces of information: a CG described using the GLARE language [9], a BMK described by means of the $\mathcal{S} C I F F$ language [1], and a set of data about the patient (the "Patient DB"). The Patient DB includes the patient data (a description of the patient and her medical situation); and the actual execution (a list of the medical actions executed on that patient).

The conformance is evaluated by means of two different modules, and their output is combined to provide possible explanations. The first module is the $C G$ Conformance Module. It compares the observed execution with the "ideal" execution, to spot possible differences: $(i)$ executed actions not envisaged by the $\mathrm{CG}$, and $(\mathrm{ii})$ actions foreseen by the CG but not observed in the execution. The ideal execution is obtained through the GLARE system, by applying specific patient data to the generic CG description.

The second module, the BMK Conformance Module, tests the conformance of the actual execution w.r.t. the BMK, expressed using the $\mathcal{S} C I F F$ formalism. The $\mathcal{S}$ CIFF Proof Procedure generates expectations about the events, and automatically checks if such expectations are fulfilled or not. Besides a yes/no answer, the $\mathcal{S}$ CIFF Proof Procedure provides also explanations, in terms of the expectations about the happened/not happened events.

Finally, the Explanation Module combines the outputs of the two previous modules: a list of discrepancies (observed/not observed medical actions) is given to the physician, together with expectations that might justify the presence/absence of the observed discrepancies.

\section{Related Works}

Several proposals for representing CGs have been made: in $[6,8]$ the interested reader can find surveys and comparisons of the many formalisms. Recently, there has been a growing interest in declarative paradigms for modeling process models. In [5] the declarative language CIGDEC for clinical guidelines is presented. 
CIGDEC lets the user to define constraints that must hold among activities execution, but integration with procedural specifications are not considered. In [3] the authors integrate the domain knowledge within an adaptive Process Management System (PMS), by means of semantic constraints. A generic criterion for semantic correctness of processes is given. However, unlike $\mathcal{S} C I F F$ semantic constraints can not take into account contexts, time, locations and data in general.

In [7] the problem of augmenting CG rules with collateral medical information was first pointed out. More recently, in Protocure and Protocure II EU projects there has been an extensive attempt to couple CGs and BMK. CGS are modeled using Asbru, while the BMK is given as a set of formulas in future-time LTL. The theorem prover KIV is used to perform quality checks [2], focussing on properties of CG "per se", and ignoring the conformance dimension.

Acknowledgements. This work has been partially supported by the FIRB project TOCAI.it (RBNE05BFRK) and by the Italian MIUR PRIN 2007 project No. 20077 WWCR8.

\section{References}

1. M. Alberti, F. Chesani, M. Gavanelli, E. Lamma, P. Mello, and P. Torroni. Verifiable agent interaction in abductive logic programming: the SCIFF framework. ACM Transactions on Computational Logics, 9(4), 2008.

2. A. Hommersom, P. Groot, P.J.F. Lucas, M. Balser, and J. Schmitt. Verification of medical guidelines using background knowledge in task networks. Knowledge and Data Engineering, IEEE Transactions on, 19(6):832-846, June 2007.

3. L. T. Ly, S. Rinderle, and P. Dadam. Integration and verification of semantic constraints in adaptive process management systems. Data Knowl. Eng., 64(1):323, 2008 .

4. Field MJ and Lohr KN, editors. Clinical Practice Guidelines: Directions for a New Program. Institute of Medicine, Washington, DC, National Academy Press, 1990.

5. N. Mulyar, M. Pesic, W. M. P. van der Aalst, and M. Peleg. Declarative and procedural approaches for modelling clinical guidelines: Addressing flexibility issues. In BPM Workshops, volume 4928 of LNCS, pages 335-346. Springer, 2007.

6. M. Peleg, S. Tu, J. Bury, P. Ciccarese, N. Jones, J. Fox, R. A. Greenes, R. Hall, P.D. Johnson, N. Jones, A. Kumar, S. Miksch, S. Quaglini, A. Seyfang, E. H. Shortliffe, and M. Stefanelli. Comparing computer-interpretable guideline models: A casestudy approach. JAMIA, 10(1), 2003.

7. R. N. Shiffman. Representation of clinical practice guidelines in conventional and augmented decision tables. J Am Med Inform Assoc, 4(5):382-393, Sep-Oct 1997.

8. A. Ten Teije, S. Miksch, and P. Lucas, editors. Computer-based Medical Guidelines and Protocols: A Primer and Current Trends, volume 139 of Studies in Health Technology and Informatics. IOS Press, Amsterdam, July 2008.

9. P. Terenziani, S. Montani, A. Bottrighi, G. Molino, and M. Torchio. Applying Artificial Intelligence to Clinical Guidelines: the GLARE Approach. Volume 139 of Teije et al. [8], July 2008. 